\title{
The impact of DSR index on online purchase decision: Based on price adjustment effect
}

\author{
Mei Yiran ${ }^{1, a}$ \\ ${ }^{1}$ Department of accounting, East China University of Science and Technology, Shanghai, China \\ ameiyiran@163.com \\ ${ }^{*}$ Corresponding author
}

Keywords: Network reputation system; DSR score; product price; moderating effect

\begin{abstract}
Taobao Store Dynamic Rating (DSR) includes three indicators: truthful descriptions, service attitudes, logistics services. How do these indicators impact on customers online purchasing decision-making? How will the price of products affect these three indicators on the online purchasing decision-making? In view of the three indicators of DSR, this paper collects and summarizes the information of Taobao stores and the massive purchase data of consumers, and studies how these factors affect online purchasing decision-making.
\end{abstract}

\section{DSR 指标对在线购买决策的影响探究：基于价格的调节效应}

\author{
梅怡然, a \\ 1华东理工大学会计学系, 上海, 中国 \\ ameiyiran@163.com \\ “通讯作者
}

关键词：网络信誉体系; DSR评分；产品价格；调节效应

中文摘要. 淘宝店铺动态评分(DSR)包括三个指标: 如实描述、服务态度、物流服务, 这三个 指标对淘宝客户在线购买决策的影响具体是怎么样的呢? 产品价格高低又将对这三个指标影 响在线购买决策带来怎么样的变化呢? 针对DSR的三个指标, 本文对淘宝店铺信息以及消费 者海量购买数据进行采集汇总，研究这些因素如何对在线购买决策产生影响。

\section{1. 引言}

2017 年的天猫双 11 狂欢节好像还在眼前, 转眼又要迎来 2018 年的双 11。据阿里巴巴集 团公布的数据显示, 2017 年双 11 当天的全天总交易额突破 1682 亿元，而就在此之前的 2016 年和 2015 年全天总交易额则分别是 1207 亿元和 912.17 亿元。短短三年间, 天猫双 11 的规 模增长如此迅速, 可见网上购物的发展越来越普及, 也越来越大势所趋。在互联网购物背景 下, 影响消费者在线购买决策的因素成为了消费者行为研究中的一个热门话题。这些因素直 接或者间接地影响到顾客的购物体验, 同时高速发展的电子商务平台需要建立有效的网络信 誉评价体系来促成买卖双方的交易。

对于网购商品而言，价格与销量之间的关系并没有传统市场中的商品这么明显。目前已 有的研究对此还没有得出统一的结果, 有的研究发现网购商品的价格与销量强相关, 也有的 研究发现网购商品的价格与销量弱相关, 甚者在有的研究中这两者之间不相关。经济学的研 究为这一现象提供了一定的解释。在市场运作中, 信息起着至关重要的作用, 信息不对称可 
能会导致“赢家的诅咒”、卖家操纵市场、市场萎缩乃至消失等一系列严重的问题。合理的信 誉体系作为电子商务平台提供信息的重要组成部分, 能促使理性人抗拒短期利益诱惑、诚实 交易，从而有效防范或规避道德风险或者逆向选择这类现象的发生。

阿里巴巴提供了一个范围广泛的虚拟网络平台, 在这个平台上买家与卖家进行大量的交 易。而我们知道, 网络虚拟条件下的非经常性交易更需要信誉体系的参与, 在保证交易顺利 进行的同时, 无形中也能维系平台的长期运营。海量的信息、全球化的触角、虚拟交流的局 限性以及复杂的网络技术给网络信誉体系的建立提出了许多新的问题。在传统的线下交易中, 买家主要凭借本身已经对卖家存在的认知以及对直接接触交易商品的感受来做出购买决策, 此外, 还可以通过认识熟悉的人得到有关卖家的信誉等信息。这种口碑传递的信誉在线下交 易中常见且易于接受。但是, 在线上交易中, 平台提供了海量的商品以及商家店铺供消费者 选择, 导致通常情况下的交易双方都是第一次线上接触, 卖家的信誉评分过程看似透明, 实 则也是一个“黑箱”。那么, 消费者是否会相信淘宝店铺的 DRS 评分呢? 淘宝店铺的 DSR 评 分高低是否会影响消费者做出购买决策呢? 是否在商品价格更高的时候, 越高的 DSR 评分越 能促进消费者购买决定的做出呢? 更进一步地, DSR 具体包括 3 个指标：如实描述、服务态 度、物流服务。这三个指标在消费者做出购买决策的过程中起到的作用是否一致呢？

本文选择淘宝网这一平台作为研究对象。在网络购物中, 淘宝网作为中国最大的 $\mathrm{C} 2 \mathrm{C}$ 线 上交易平台, 有着非常重要的市场地位, 因而这一研究对象非常具有代表性。另外, 淘宝网 上的买卖双方参与者数量极大, 更接近于竞争性市场, 大部分卖家的信誉主要通过网络渠道 建立; 相比之下, $\mathrm{B} 2 \mathrm{C}$ 平台上大部分卖家则可以通过线下市场的信誉度来打开线上市场。本 文将以信誉体系为基础, 重点关注 DSR 的 3 个指标以及这 3 个指标在价格的调节作用下如何 对消费者的购买决策起到影响，探讨它们之间的因果关系和调节效应。

本文更加详细地探究了淘宝店铺的个体信誉下的子指标（如实描述、服务态度、物流服 务）对消费者决策的作用机制, 最终的结论将有利于引导卖家更加关注其中最重要的指标, 并针对性地进行个体信誉管理。

\section{2. 文献综述与研究假设}

\section{1 网络信誉体系}

网络信誉体系被世界上电子商务企业所普遍采用, 用以衡量卖家的诚信和服务水平。美 国主要的电子商务平台 eBay 与淘宝的信誉体系设定比较相近, 都是由信用等级、DSR 评分(动 态店铺评分) 以及文字评论三部分组成的。信用等级是指用户在线上交易后给出的评价, 如好 评、中评或者差评。好评计 +1 分, 中评计 0 分, 差评计 -1 分, 卖家的累计分数超过一定限度, 就可以获得相应的称号。例如 eBay 上卖家可以获得类似 Top-rated seller 的的称号, 而淘宝上 的卖家可以获得心、钻、冠的等级。目前, 对电子商务信誉体系的研究主要集中在对信用等 级体系的研究上, 而较少涉及对 DSR 评分体系或者文字评论体系的研究, 原因在于 DSR 评 分体系是一个相对信用等级体系来说较新的体系, 而文字评论体系在量化上面存在着一定的 困难。然而, DSR 评分体系和文字评论体系这两种较为年轻的体系对于消费者来说包含着更 多的信息量, 因为 DSR 评分体系从产品质量、服务质量以及物流服务质量等方面给消费者展 示卖家近一段时间内收到的评分的动态平均值, 包含比信用等级体系更为明确的和更新的信 息, 而文字评论信息比评分更为具体生动。与 DSR 评分体系和文字评论体系相比, 传统的信 用等级体系的好评率一般都在 $99 \%$ 以上, 对卖家的区分作用不如前两者大。因此, 对 DSR 评 分体系和文字评论体系的更多研究是十分必要的。 
表1＼cjkstart信誉体系对商品销售量、成交率及价格影响的文献总结

\begin{tabular}{|c|c|c|}
\hline 作者 & 研究对象 & 主要研究结论 \\
\hline Resnick (2002) & MP3、娃娃玩具 & $\begin{array}{c}\text { 好评与成交成功率呈正相关关系, 但是好评和 } \\
\text { 差评对价格都没有显著影响 }\end{array}$ \\
\hline Melnik,Alm（2002） & 收藏性金币 & $\begin{array}{c}\text { 产品信用评分越高, 成交价格就越高, 但是影 } \\
\text { 响幅度微乎其微 }\end{array}$ \\
\hline Dewan,Hsu（2004） & 邮票 & $\begin{array}{c}\text { 累计好评数量对拍卖的成交率以及最终得成交 } \\
\text { 价格产生显著的正影响 }\end{array}$ \\
\hline Eaton $(2005)$ & 吉他 & $\begin{array}{c}\text { 差评会对成交率产生显著的负面影响, 信用评 } \\
\text { 分对价格无显著影响 }\end{array}$ \\
\hline Melnik,Alm（2005） & 收藏性金币 & $\begin{array}{c}\text { 异质化程度高的金币信用评价对价格的影响更 } \\
\text { 显著, 对于经过第三方认证的金币, 信用评分 } \\
\text { 对价格的影响不显著 }\end{array}$ \\
\hline 周黎安等（2006） & 多种产品 & $\begin{array}{c}\text { 信誉分数与好评率与价格和成交率都呈正相关 } \\
\text { 关系 }\end{array}$ \\
\hline Jin,Kato (2006) & 棒球、卡片 & $\begin{array}{c}\text { 信用评分对成交率有正向影响, 尤其是对没有 } \\
\text { 专业评级的卡片影响特别显著 }\end{array}$ \\
\hline Houser,Wooders(2006) & 电脑处理器 & 好评数与价格呈正相关关系 \\
\hline 李维安等 (2007) & 魔兽世界点卡 & 信用等级会对销售量产生显著的正向影响 \\
\hline WU Wei-fang等（2008） & 数码相机 & $\begin{array}{l}\text { 基于淘宝的研究, 好评数与更大的销量显著相 } \\
\text { 关, 更多的好评数与更低的成交价格相联系 }\end{array}$ \\
\hline Cabral 等（2010） & 多种产品 & $\begin{array}{l}\text { 差评会对交易量产生显著的负面影响, 其 } \\
\text { 中第一个差评产生的影响最为明显 }\end{array}$ \\
\hline Lei (2011) & Gmail 邀请 & $\begin{array}{l}\text { 更高的信誉等级对成交率和价格都有显著 } \\
\text { 的正向影响 }\end{array}$ \\
\hline
\end{tabular}

\section{2 声誉与购买意愿}

现有理论认为, 如果在购买之前无法观察到产品的质量, 消费者可能会通过产品价格、 广告或商家的声誉来进行推断。这些“信号”和产品真实质量之间的关系已经得到了验证。比 如, Nelson(1970)发现通常只有高质量产品的卖家更能并且更愿意承受高额的广告费, 因为高 质量的产品更能带来买家的重复购买。而 Klein 和 Leffler(1981)则认为高质量产品卖家的高价 格会产生持续的租金收入，这会促使卖家在长期内提供良好质量的产品而不是短期内的欺骗 与消失。同样地, Shapiro (1983)研究发现在过去提供良好质量产品的卖家会更加倾向于去维 持他们的信誉, 因为良好的信誉会给他们未来的高价格与高收益打下基础。此外, Ginger Zhe Jin and Andrew Kato (2006) 发现在互联网拍卖中, 在线卖家声誉对于识别诚信卖家是有效的, 并且这些高声望卖家的目标客户都定位为有经验的买家。

毫无疑问, 在线购买的买家更愿意购买高质量的商品。考虑到以上研究得出的高质量的 卖家更倾向于维持声誉的结论, 我们认为更高的 DSR 评分更能促进消费者的购买决策, 因此 得到假设 $\mathrm{H}_{1}$ :

$\mathrm{H}_{1 \mathrm{a}}$ ：DSR 评分中的“如实描述”得分越高, 越能促进消费者购买产品;

$\mathrm{H}_{1 b}$ ：DSR 评分中的“服务态度”得分越高，越能促进消费者购买产品;

$\mathrm{H}_{1 \mathrm{c}}$ ：DSR 评分中的“物流服务”得分越高, 越能促进消费者购买产品。

\section{3 声誉、价格与购买意愿}

钟梅 (2013) 认为: 个体信誉对价格溢价的影响不显著,即使当消费者感知到商家的个体 信誉较高时也不愿意支付较高的价格。笔者在对比淘宝中同类商品定价时也发现, 商品定价与 
个体信誉高低没有必然关系,有些信誉高的商家甚至定价更低。这可能是因为在显著同业竞争 激励的环境中,每一个商品都可能有成百上千的卖家在同时出售,稍高的价格可能导致买家的 流失,转向别的卖家。再加上现在的信誉炒作行为频繁被曝光,个人信誉在也备受质疑,消费者 不能感到高个体信誉带来的质量保障,因此也不愿意支付更高的价格。淘宝店铺中 DSR 得分 较好的数量并不在少数, 消费者并不仅仅依靠 DSR 的 3 个指标做出购买决策, 当商品价格较 高时, 消费者应当更倾向于选择 DSR 得分高的淘宝店铺。因此, 本文认为, 商品的价格会对 DSR 指标与购买决策之间的关系产生调节作用，由此得出假设 $\mathrm{H}_{2}$ :

$\mathrm{H}_{2 \mathrm{a}}$ ：商品价格会对 DSR 指标与购买决策之间的关系产生调节作用。其中，高价格商品 所在店铺的“描述相符”指标对消费者购买决策的作用更明显。

$\mathrm{H}_{2 \mathrm{~b}}$ ：商品价格会对 DSR 指标与购买决策之间的关系产生调节作用。其中，高价格商品 所在店铺的“服务态度”指标对消费者购买决策的作用更明显。

$\mathrm{H}_{2 \mathrm{c}}$ ：商品价格会对 DSR 指标与购买决策之间的关系产生调节作用。其中，高价格商品 所在店铺的“物流服务”指标对消费者购买决策的作用更明显。

\section{3. 模型与变量}

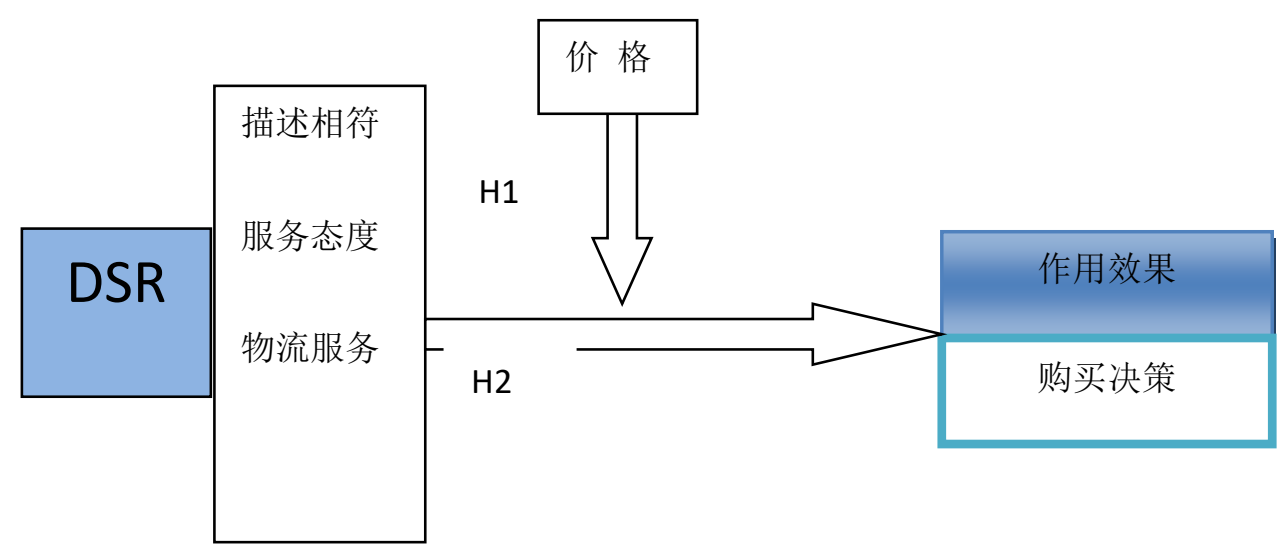

图1 概念模型图

本文的研究模型如上图所示, 本文研究的被解释变量为购买意愿, 其中, 若消费者进入 商品页面后购买了商品, 则值为 1 , 若未购买商品, 则值为 0 。解释变量为淘宝店铺的动态评 分(DSR)，包括“描述相符”、“服务态度”和“物流速度”这三个指标。本文认为这 3 个指标对消 费者的购买决策产生影响。控制变量包括产品价格、产品近 30 天销量、信用等级、好评率、 开店时长、评论次数以及评论长度等。以上变量的分类、名称及测度等详见表 2 。

表2模型变量测度指标

\begin{tabular}{|c|c|c|}
\hline 变量类型 & 变量名称 & 变量测量 \\
\hline 因变量 & 是否购买 & 买家在点击商品页面后是否进行购买 \\
\hline \multirow{4}{*}{ 自变量 } & 描述相符 & \multirow{2}{*}{ 提取自淘宝界面, 表示 (同行业平均分-店铺得 } \\
& 分) / (同行业平均分-同行业店铺最低得分) \\
\cline { 2 - 3 } & 物流服务 & 商品30天内的平均成交价格 (单位: 千元) \\
\hline \multirow{5}{*}{ 控制变量 } & 产品价格 & 该商品在淘宝上近30天的累计成交量 \\
\cline { 2 - 3 } & 近30天销量 & 卖家在淘宝网中获得的信用等级 \\
\cline { 2 - 3 } & 信用等级 & 卖家得到好评的次数/卖家得到评价的次数 \\
\cline { 2 - 3 } & 好评率 & 卖家在淘宝上开店的时间 (单位: 年) \\
\cline { 2 - 3 } & 开店时长 & 买家在单品页面的评论次数 \\
\cline { 2 - 3 } & 评论次数 & \\
\hline
\end{tabular}




\section{Reference}

[1] Resnick,P. and Zeckhauser, R. Trust among Strangers in Internet Transactions: Empirical Analysis of eBay's Reputation System [J]. Advances in Applied Microeconomics,2002.11:127-157.

[2] Melnik M and Alm J. Does a Seller's Ecommerce Reputation Matter? Evidence from eBay Auctions[J].The Journal of Industrial Economics, 2002.50(3):337-349.

[3] Dewan S and Hsu V. Adverse Selection in Electronic Markets: Evidence from Online Stamp Autions[J]. The Journal of Industrial Economics,2004,52(4):497-516.

[4] Eaton D H. Valuing Information: Evidence from Guitar Auctions on eBay[J]. Journal of Applied Economics and Policy.2005,24(1):1-19.

[5] Melnik M and Alm J. Seller Reputation, Information Signals, and Prices for Hetergeneous Coins on eBay[J].Southern Economic Journal, 2005,72(2):305-328.

[6] Zhou Li' an, Zhang Weiying, Gu Quanlin and Shen Yi. The Value of Reputation :Evidence from Online Auctions [J], Economic Research Journal. 2006(12):81-91+124.

[7] Jin G Z and Kato A. Price, Quality, and Reputation :Evidence From an Online Field Experiment[J]. The RAND Jounal of Economics, 2006,37(4):983-1005.

[8] Houser D and Wooders J. Reputation in Auctions: Theory, and Evidence from eBay[J]. Journal of Economics\& Management Strategy, 2006,15(2):353-369.

[9] Li Wei'an, Wu Desheng and Xu Hao. Reputation in China's Online Auction Market: Evidence from the Taobao Website [J]. Nankai Business Review, 2007(05):36-46.

[10] Wu W F and Qiang Ye. The Impact of Reputation System on C2C Online Sales: Evidence from China[M]. Management Science and Eneineering 15th Annual Conference Proceedings,2008:523-528.

[11]Cabral L and Hortacsu A. The Dynamics of Seller Reputation: Evidence from E-Bay [J].The Journal of Industrial Economics,2010.58(1):54-78.

[12]Lei Q. Financial Value of Reputation :Evidence from the eBay Auctions of Gmail Invitations [J]. The Journal of Industrial Economics, 2011.59(3):422-546.

[13]Nelson P. "Information and Consumer Behavior."[J]. Journal of Political Economy, Vol. 78 (1970):311-329.

[14]Klein B. and Leffler K B. The Role of Market Forces in Assuring Contractual Performance.[J] Journal of Political Economy, Vol. 89(1981): 615-641.

[15] Shapiro C. Premiums for High Quality Products as Returns to Reputation.[J]Quarterly Journal of Economics, Vol.98(1983): 659-680.

[16]Zhong Mei. The Empirical Analysis and Measures of Collective Reputation Impact on Customer Decision-making [D]. Southwestern University of Finance and Economics,2013. 